\title{
Systems with the integer rounding property in normal monomial subrings
}

\author{
LUIS A. DUPONT ${ }^{1}$, CARLOS RENTERÍA-MÁRQUEZ ${ }^{2}$ and RAFAEL H. VILLARREAL ${ }^{1}$ \\ ${ }^{1}$ Departamento de Matemáticas, Centro de Investigación y de Estudios Avanzados del IPN, \\ Apartado Postal 14-740, 07000 Mexico City, D.F. \\ ${ }^{2}$ Departamento de Matemáticas, Escuela Superior de Física y Matemáticas, Instituto Politécnico Nacional, \\ Unidad Adolfo López Mateos, Edif. 9, 07738 México City, D.F.
}

Manuscript received on September 9, 2009; accepted for publication on May 5, 2010

\begin{abstract}
Let $\mathcal{C}$ be a clutter and let $A$ be its incidence matrix. If the linear system $x \geq 0 ; x A \leq \mathbf{1}$ has the integer rounding property, we give a description of the canonical module and the $a$-invariant of certain normal subrings associated to $\mathcal{C}$. If the clutter is a connected graph, we describe when the aforementioned linear system has the integer rounding property in combinatorial and algebraic terms using graph theory and the theory of Rees algebras. As a consequence we show that the extended Rees algebra of the edge ideal of a bipartite graph is Gorenstein if and only if the graph is unmixed.
\end{abstract}

Key words: canonical module, $a$-invariant, normal ideal, perfect graph, maximal cliques, Rees algebra, Ehrhart ring, integer rounding property.

\section{INTRODUCTION}

A clutter $\mathcal{C}$ with finite vertex set $X$ is a family of subsets of $X$ called edges, and none of which is included in another. The set of vertices and edges of $\mathcal{C}$ are denoted by $V(\mathcal{C})$ and $E(\mathcal{C})$, respectively. A basic example of a clutter is a graph.

Let $\mathcal{C}$ be a clutter with finite vertex set $X=\left\{x_{1}, \ldots, x_{n}\right\}$. We shall always assume that $\mathcal{C}$ has no isolated vertices, i.e., each vertex occurs in at least one edge. Let $f_{1}, \ldots, f_{q}$ be the edges of $\mathcal{C}$, and let $v_{k}=\sum_{x_{i} \in f_{k}} e_{i}$ be the characteristic vector of $f_{k}$, where $e_{i}$ is the $i$ th unit vector in $\mathbb{R}^{n}$. The incidence matrix $A$ of $\mathcal{C}$ is the $n \times q$ matrix with column vectors $v_{1}, \ldots, v_{q}$. If $a=\left(a_{i}\right)$ and $c=\left(c_{i}\right)$ are vectors in $\mathbb{R}^{n}$, then $a \leq c$ means that $a_{i} \leq c_{i}$ for all $i$. Thus, $a \geq 0$ means that $a_{i} \geq 0$ for all $i$. The system $x \geq 0 ; x A \leq \mathbf{1}$ has the integer rounding property if

$$
\lceil\min \{\langle y, \mathbf{1}\rangle \mid y \geq 0 ; A y \geq \alpha\}\rceil=\min \left\{\langle y, \mathbf{1}\rangle \mid A y \geq \alpha ; y \in \mathbb{N}^{q}\right\}
$$

AMS Classification: Primary 13B22; Secondary 13H10, 13F20, 52B20.

Correspondence to: Rafael H. Villarreal

E-mail: vila@math.cinvestav.mx 
for each integral vector $\alpha$ for which $\min \{\langle y, \mathbf{1}\rangle \mid y \geq 0 ; A y \geq \alpha\}$ is finite. Here $\mathbf{1}$ denotes the vector with all its entries equal to 1 , and $\langle$,$\rangle denotes the standard inner product. For a thorough study of this property$ see (Schrijver 1986).

Let $R=K\left[x_{1}, \ldots, x_{n}\right]$ be a polynomial ring over a field $K$, and let $w_{1}, \ldots, w_{r}$ be the set of all integral vectors $\alpha$ such that $0 \leq \alpha \leq v_{i}$ for some $i$. We will examine the integer rounding property using the monomial subring:

$$
S=K\left[x^{w_{1}} t, \ldots, x^{w_{r}} t\right] \subset R[t],
$$

where $t$ is a new variable. As usual, we use the notation $x^{a}:=x_{1}^{a_{1}} \cdots x_{n}^{a_{n}}$, where $a=\left(a_{1}, \ldots, a_{n}\right) \in \mathbb{N}^{n}$. The subring $S$ is called normal if $S$ is integrally closed, i.e., $S=\bar{S}$, where $\bar{S}$ is the integral closure of $S$ in its field of fractions; see (Vasconcelos 2005).

The contents of this paper are as follows. One of the results in (Brennan et al. 2008) shows that $S$ is normal if and only if the system $x \geq 0 ; x A \leq \mathbf{1}$ has the integer rounding property (see Theorem 2.1). As a consequence, we show that if all edges of $\mathcal{C}$ have the same number of elements and either linear system $x \geq 0 ; x A \leq \mathbf{1}$ or $x \geq 0 ; x A \geq \mathbf{1}$ has the integer rounding property, then, the subring $K\left[x^{v_{1}} t, \ldots, x^{v_{q}} t\right]$ is normal (see Corollary 2.4).

Let $G$ be a connected graph and let $A$ be its incidence matrix. The main results of Section 3 show that the following conditions are equivalent (see Theorems 3.2 and 3.3):

(a) $x \geq 0 ; x A \leq \mathbf{1}$ has the integer rounding property.

(b) $x \geq 0 ; x A \geq \mathbf{1}$ has the integer rounding property (see Definition 2.2).

(c) $R\left[x^{v_{1}} t, \ldots, x^{v_{q}} t\right]$ is normal, where $v_{1}, \ldots, v_{q}$ are the column vectors of $A$.

(d) $K\left[x^{v_{1}} t, \ldots, x^{v_{q}} t\right]$ is normal.

(e) $K\left[t, x_{1} t, \ldots, x_{n} t, x^{v_{1}} t, \ldots, x^{v_{q}} t\right]$ is normal.

(f) The induced subgraph of the vertices of any two vertex disjoint odd cycles of $G$ is connected.

The most interesting part of this result is the equivalence between (a), which is a linear optimization property, and (f), which is a graph theoretical property. Edge ideals are defined in Section 2. We prove that the ring in (e) is isomorphic to the extended Rees algebra of the edge ideal of $G$ (see Proposition 3.1). If $G$ is bipartite and $I=I(G)$ is its edge ideal, we are able to prove that the extended Rees algebra of $I$ is a Gorenstein standard $K$-algebra if and only if $G$ is unmixed (see Corollary 4.3). If we work in the more general context of clutters, none of the conditions (a) to (e) are equivalent. Some of these conditions are equivalent under certain assumptions (Gitler et al. 2009).

In Section 4 we introduce the canonical module and the $a$-invariant of $S$. This invariant plays a key role in the theory of Hilbert functions (Bruns and Herzog 1997). There are some methods, based on combinatorial optimization, that have been used to study canonical modules of edge subrings of bipartite graphs (Valencia and Villarreal 2003). Our approach to study canonical modules is inspired by these 
methods. If $S$ is a normal domain, we express the canonical module of $S$ and its $a$-invariant in terms of the vertices of the polytope

$$
\{x \mid x \geq 0 ; x A \leq \mathbf{1}\}
$$

(see Theorem 4.1). We are able to give an explicit description of the canonical module of $S$ and its $a$-invariant when $\mathcal{C}$ is the clutter of maximal cliques of a perfect graph (Theorem 4.2).

For unexplained terminology and notation on commutative algebra and integer programming, we refer to (Bruns and Herzog 1997, Vasconcelos 2005, Villarreal 2001) and (Schrijver 1986), respectively.

\section{INTEGER ROUNDING AND NORMALITY}

We continue using the definitions and terms from the Introduction. In what follows, $\mathbb{N}$ denotes the set of non-negative integers and $\mathbb{R}_{+}$denotes the set of non-negative real numbers. Let $\mathcal{A} \subset \mathbb{Z}^{n}$. The convex hull of $\mathcal{A}$ is denoted by $\operatorname{conv}(\mathcal{A})$, and the cone generated by $\mathcal{A}$ is denoted by $\mathbb{R}_{+} \mathcal{A}$.

THEOREM 2.1 (Brennan et al. 2008). Let $\mathcal{C}$ be a clutter and let $v_{1}, \ldots, v_{q}$ be the columns of the incidence matrix $A$ of $\mathcal{C}$. If $w_{1}, \ldots, w_{r}$ is the set of all $\alpha \in \mathbb{N}^{n}$ such that $\alpha \leq v_{i}$ for some $i$, then the system $x \geq 0 ; x A \leq \mathbf{1}$ has the integer rounding property if and only if the subring $K\left[x^{w_{1}} t, \ldots, x^{w_{r}} t\right]$ is normal.

Next we give an application of this result, but first we need to introduce some more terminology and notation. We have already defined in the Introduction when the linear system $x \geq 0 ; x A \leq \mathbf{1}$ has the integer rounding property. The following is a dual notion.

Definition 2.2. Let $A$ be a matrix with entries in $\mathbb{N}$. The system $x \geq 0 ; x A \geq \mathbf{1}$ has the integer rounding property if

$$
\max \left\{\langle y, \mathbf{1}\rangle \mid A y \leq w ; y \in \mathbb{N}^{q}\right\}=\lfloor\max \{\langle y, \mathbf{1}\rangle \mid y \geq 0 ; A y \leq w\}\rfloor
$$

for each integral vector $w$ for which the right hand side is finite.

Let $\mathcal{A}=\left\{v_{1}, \ldots, v_{q}\right\}$ be a set of points in $\mathbb{N}^{n}$, let $P$ be the convex hull of $\mathcal{A}$, and let $R=K\left[x_{1}, \ldots, x_{n}\right]$ be a polynomial ring over a field $K$. The Ehrhart ring of the lattice polytope $P$ is the monomial subring

$$
A(P)=K\left[\left\{x^{a} t^{i} \mid a \in \mathbb{Z}^{n} \cap i P ; i \in \mathbb{N}\right\}\right] \subset R[t],
$$

where $t$ is a new variable. A nice property of $A(P)$ is that it is always a normal domain (Bruns and Herzog 1997). Let $\mathcal{C}$ be a clutter with vertex set $X=\left\{x_{1}, \ldots, x_{n}\right\}$ and edge set $E(\mathcal{C})$. For use below recall that $\mathcal{C}$ is called uniform if all its edges have the same number of elements. The edge ideal of $\mathcal{C}$, denoted by $I(\mathcal{C})$, is the ideal of $R$ generated by all monomials $\prod_{x_{i} \in e} x_{i}=x_{e}$ such that $e \in E(\mathcal{C})$. The Rees algebra of $I=I(\mathcal{C})$, denoted by $R[I t]$, is given by

$$
R[I t]:=R \oplus I t \oplus \cdots \oplus I^{i} t^{i} \oplus \cdots \subset R[t],
$$

see (Vasconcelos 2005, Chapter 1) for a nice presentation of Rees algebras.

The next result holds for arbitrary monomial ideals (not necessarily square-free). This is the only place in the paper where a result is stated for arbitrary monomial ideals. 
THEOREM 2.3 (Dupont and Villarreal 2010). Let $I=\left(x^{v_{1}}, \ldots, x^{v_{q}}\right) \subset R$ be a monomial ideal, and let $A$ be the matrix with column vectors $v_{1}, \ldots, v_{q}$. Then, the system $x \geq 0 ; x A \geq \mathbf{1}$ has the integer rounding property if and only if $R[I t]$ is normal.

COROllary 2.4. Let $\mathcal{C}$ be a uniform clutter, let $A$ be its incidence matrix, and let $v_{1}, \ldots, v_{q}$ be the columns of $A$. If either system $x \geq 0 ; x A \leq \mathbf{1}$ or $x \geq 0 ; x A \geq \mathbf{1}$ has the integer rounding property and $P=\operatorname{conv}\left(v_{1}, \ldots, v_{q}\right)$, then

$$
K\left[x^{v_{1}} t, \ldots, x^{v_{q}} t\right]=A(P) .
$$

Proof. In general, the subring $K\left[x^{v_{1}} t, \ldots, x^{\left.v_{q} t\right]}\right.$ is contained in $A(P)$. Assume that $x \geq 0 ; x A \leq \mathbf{1}$ has the integer rounding property, and that every edge of $\mathcal{C}$ has $d$ elements. Let $w_{1}, \ldots, w_{r}$ be the set of all $\alpha \in \mathbb{N}^{n}$ such that $\alpha \leq v_{i}$ for some $i$. Then by Theorem 2.1, the subring $K\left[x^{w_{1}} t, \ldots, x^{w_{r}} t\right]$ is normal. Using that $v_{1}, \ldots, v_{q}$ is the set of $w_{i}$, with $\left|w_{i}\right|=d$, it is not hard to see that $A(P)$ is contained in $K\left[x^{v_{1}} t, \ldots, x^{v_{q}} t\right]$.

Assume that $x \geq 0 ; x A \geq \mathbf{1}$ has the integer rounding property. Let $I=I(\mathcal{C})$ be the edge ideal of $\mathcal{C}$, and let $R[I t]$ be its Rees algebra. By Theorem 2.3, $R[I t]$ is a normal domain. Since the clutter $\mathcal{C}$ is uniform, the required equality follows at once (Escobar et al. 2003, Theorem 3.15).

The converse of Corollary 2.4 fails as the following example shows.

EXAMPLE 2.5. Let $\mathcal{C}$ be the uniform clutter with vertex set $X=\left\{x_{1}, \ldots, x_{8}\right\}$ and edge set

$$
E(\mathcal{C})=\left\{\left\{x_{3}, x_{4}, x_{6}, x_{8}\right\},\left\{x_{2}, x_{5}, x_{6}, x_{7}\right\},\left\{x_{1}, x_{4}, x_{5}, x_{8}\right\},\left\{x_{1}, x_{2}, x_{3}, x_{8}\right\}\right\}
$$

The characteristic vectors of the edges of $\mathcal{C}$ are

$$
\begin{array}{ll}
v_{1}=(0,0,1,1,0,1,0,1), & v_{2}=(0,1,0,0,1,1,1,0), \\
v_{3}=(1,0,0,1,1,0,0,1), & v_{4}=(1,1,1,0,0,0,0,1) .
\end{array}
$$

Let $A$ be the incidence matrix of $\mathcal{C}$ with column vectors $v_{1}, \ldots, v_{4}$, and let $P$ be the convex hull of $\left\{v_{1}, \ldots, v_{4}\right\}$. It is not hard to verify that the set

$$
\left\{\left(v_{1}, 1\right),\left(v_{2}, 1\right),\left(v_{3}, 1\right),\left(v_{4}, 1\right)\right\}
$$

is a Hilbert basis in the sense of (Schrijver 1986). Therefore, we have the equality

$$
K\left[x^{v_{1}} t, x^{v_{2}} t, x^{v_{3}} t, x^{v_{4}} t\right]=A(P) .
$$

Using Theorem 2.3 and (Brennan et al. 2008, Theorem 2.12) it is seen that none of the two systems $x \geq 0 ; x A \leq \mathbf{1}$ and $x \geq 0 ; x A \geq \mathbf{1}$ have the integer rounding property.

\section{INCIDENCE MATRICES OF GRAPHS}

Let $G$ be a connected graph with vertex set $X=\left\{x_{1}, \ldots, x_{n}\right\}$, and let $v_{1}, \ldots, v_{q}$ be the column vectors of the incidence matrix $A$ of $G$. The main result here is a purely combinatorial description of the integer 
rounding property of the system $x \geq 0 ; x A \leq 1$. Other equivalent algebraic conditions of this property will be presented.

Let $R=K\left[x_{1}, \ldots, x_{n}\right]$ be a polynomial ring over a field $K$, and let $I=I(G)$ be the edge ideal of $G$. Recall that the extended Rees algebra of $I$ is the subring

$$
R\left[I t, t^{-1}\right]:=R[I t]\left[t^{-1}\right] \subset R\left[t, t^{-1}\right],
$$

where $R[I t]$ is the Rees algebra of $I$. Rees algebras of edge ideals of graphs were first studied in (Simis et al. 1994).

Proposition 3.1. $R\left[I t, t^{-1}\right] \simeq K\left[t, x_{1} t, \ldots, x_{n} t, x^{v_{1}} t, \ldots, x^{v_{q}} t\right]$.

Proof. We set $S=K\left[t, x_{1} t, \ldots, x_{n} t, x^{v_{1}} t, \ldots, x^{v_{q}} t\right]$. Note that $S$ and $R\left[I t, t^{-1}\right]$ are both integral domains of the same Krull dimension; this follows from the dimension formula given in (Sturmfels 1996, Lemma 4.2). Thus, it suffices to prove that there is an epimorphism $\bar{\psi}: S \rightarrow R\left[I t, I^{-1}\right]$ of $K$-algebras.

Let $u_{0}, u_{1}, \ldots, u_{n}, t_{1}, \ldots, t_{q}$ be a new set of variables, and let $\varphi, \psi$ be the maps of $K$-algebras defined by the diagram

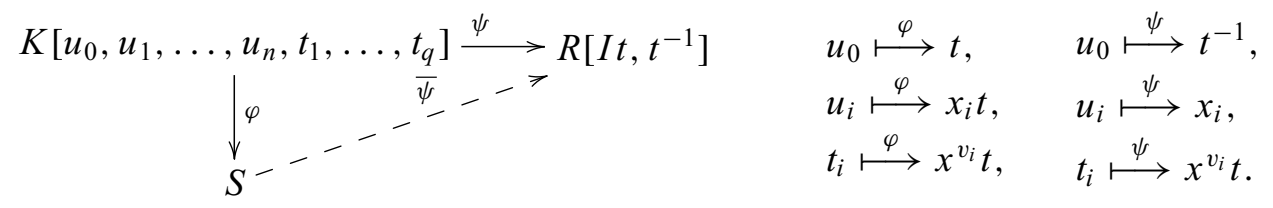

To complete the proof, we will show that there is an epimorphism $\bar{\psi}$ of $K$-algebras that makes this diagram commutative, i.e., $\psi=\bar{\psi} \varphi$. To show the existence of $\bar{\psi}$, we need only to show the inclusion $\operatorname{ker}(\varphi) \subset$ $\operatorname{ker}(\psi)$. As $\operatorname{ker}(\varphi)$ being a toric ideal is generated by binomials (Sturmfels 1996), it suffices to prove that any binomial of $\operatorname{ker}(\varphi)$ belongs to $\operatorname{ker}(\psi)$. Let

$$
f=u_{0}^{a_{0}} u_{1}^{a_{1}} \cdots u_{n}^{a_{n}} t_{1}^{b_{1}} \cdots t_{q}^{b_{q}}-u_{0}^{c_{0}} u_{1}^{c_{1}} \cdots u_{n}^{c_{n}} t_{1}^{d_{1}} \cdots t_{q}^{d_{q}}
$$

be a binomial in $\operatorname{ker}(\varphi)$. Then,

$$
t^{a_{0}}\left(x_{1} t\right)^{a_{1}} \cdots\left(x_{n} t\right)^{a_{n}}\left(x^{v_{1}} t\right)^{b_{1}} \cdots\left(x^{v_{q}} t\right)^{b_{q}}=t^{c_{0}}\left(x_{1} t\right)^{c_{1}} \cdots\left(x_{n} t\right)^{c_{n}}\left(x^{v_{1}} t\right)^{d_{1}} \cdots\left(x^{v_{q}} t\right)^{d_{q}}
$$

Taking degrees in $t$ and $\underline{x}=\left\{x_{1}, \ldots, x_{n}\right\}$, we obtain

$$
\begin{aligned}
a_{0}+\left(a_{1}+\cdots+a_{n}\right)+\left(b_{1}+\cdots+b_{q}\right) & =c_{0}+\left(c_{1}+\cdots+c_{n}\right)+\left(d_{1}+\cdots+d_{q}\right), \\
a_{1}+\cdots+a_{n}+2\left(b_{1}+\cdots+b_{q}\right) & =c_{1}+\cdots+c_{n}+2\left(d_{1}+\cdots+d_{q}\right) .
\end{aligned}
$$

Thus $-a_{0}+b_{1}+\cdots+b_{q}=-c_{0}+d_{1}+\cdots+d_{q}$, and we obtain the equality

$$
t^{-a_{0}} x_{1}^{a_{1}} \cdots x_{n}^{a_{n}}\left(x^{v_{1}} t\right)^{b_{1}} \cdots\left(x^{v_{q}} t\right)^{b_{q}}=t^{-c_{0}} x_{1}^{c_{1}} \cdots x_{n}^{c_{n}}\left(x^{v_{1}} t\right)^{d_{1}} \cdots\left(x^{v_{q}} t\right)^{d_{q}},
$$

i.e., $f \in \operatorname{ker}(\psi)$, as required. 
We come to the main result of this section.

THEOREM 3.2. Let $G$ be a connected graph and let $A$ be its incidence matrix. Then, the system

$$
x \geq 0 ; x A \leq 1
$$

has the integer rounding property if and only if the induced subgraph of the vertices of any two vertex disjoint odd cycles of $G$ is connected.

PROOF. Let $v_{1}, \ldots, v_{q}$ be the column vectors of $A$. According to (Simis et al. 1998, Theorem 1.1, cf. Villarreal 2005, Corollary 3.10), the subring $K[G t]:=K\left[x^{v_{1}} t, \ldots, x^{v_{q}} t\right]$ is normal if and only if any two vertex disjoint odd cycles of $G$ can be connected by at least one edge of $G$. Thus, we need only to show that $K[G t]$ is normal if and only the system $x \geq 0 ; x A \leq \mathbf{1}$ has the integer rounding property. Let $I=I(G)$ be the edge ideal of $G$. Since $G$ is connected, the subring $K[G t]$ is normal if and only if the Rees algebra $R[I t]$ of $I$ is normal (Simis et al. 1998, Corollary 2.8). By a result of (Herzog et al. 1991), $R[I t]$ is normal if and only if $R\left[I t, t^{-1}\right]$ is normal. By Proposition $3.1, R\left[I t, t^{-1}\right]$ is normal if and only if the subring

$$
S=K\left[t, x_{1} t, \ldots, x_{n} t, x^{v_{1}} t, \ldots, x^{v_{q}} t\right]
$$

is normal. Thus, we can apply Theorem 2.1 to conclude that $S$ is normal if and only if the system $x \geq$ $0 ; x A \leq \mathbf{1}$ has the integer rounding property.

THEOREM 3.3. Let $G$ be a connected graph and let $A$ be its incidence matrix. Then, the system $x \geq$ $0 ; x A \leq \mathbf{1}$ has the integer rounding property if and only if any of the following equivalent conditions hold

(a) $x \geq 0 ; x A \geq 1$ is a system with the integer rounding property.

(b) $R[I t]$ is a normal domain, where $I=I(G)$ is the edge ideal of $G$.

(c) $K\left[x^{v_{1}} t, \ldots, x^{v_{q}} t\right]$ is normal, where $v_{1}, \ldots, v_{q}$ are the column vectors of $A$.

(d) $K\left[t, x_{1} t, \ldots, x_{n} t, x^{v_{1}} t, \ldots, x^{v_{q}} t\right]$ is normal.

PROOF. According to Theorem 2.3, the system $x \geq 0 ; x A \geq \mathbf{1}$ has the integer rounding property if and only if the Rees algebra $R[I t]$ is normal. Thus, the result follows from the proof of Theorem 3.2.

\section{THE CANONICAL MODULE AND THE a-INVARIANT}

In this section, we give a description of the canonical module and the $a$-invariant for subrings arising from systems with the integer rounding property.

Let $\mathcal{C}$ be a clutter with vertex set $X=\left\{x_{1}, \ldots, x_{n}\right\}$, and let $v_{1}, \ldots, v_{q}$ be the columns of the incidence matrix $A$ of $\mathcal{C}$. For use below, consider the set $w_{1}, \ldots, w_{r}$ of all $\alpha \in \mathbb{N}^{n}$ such that $\alpha \leq v_{i}$ for some $i$. Let $R=K\left[x_{1}, \ldots, x_{n}\right]$ be a polynomial ring over a field $K$ and let

$$
S=K\left[x^{w_{1}} t, \ldots, x^{w_{r}} t\right] \subset R[t]
$$


be the subring of $R[t]$ generated by $x^{w_{1}} t, \ldots, x^{w_{r}} t$, where $t$ is a new variable. As $\left(w_{i}, 1\right)$ lies in the hyperplane $x_{n+1}=1$ for all $i, S$ is a standard $K$-algebra. Thus, a monomial $x^{a} t^{b}$ in $S$ has degree $b$. In what follows, we assume that $S$ has this grading. Recall that the a-invariant of $S$, denoted $a(S)$, is the degree as a rational function of the Hilbert series of $S$, see for instance (Villarreal 2001, p. 99). If $S$ is Cohen-Macaulay and $\omega_{S}$ is the canonical module of $S$, then

$$
a(S)=-\min \left\{i \mid\left(\omega_{S}\right)_{i} \neq 0\right\}
$$

see (Bruns and Herzog 1997, p. 141) and (Villarreal 2001, Proposition 4.2.3). This formula applies if $S$ is normal because normal monomial subrings are Cohen-Macaulay (Hochster 1972). If $S$ is normal, then by a formula of Danilov-Stanley, see (Bruns and Herzog 1997, Theorem 6.3.5) and (Danilov 1978), the canonical module of $S$ is the ideal given by

$$
\omega_{S}=\left(\left\{x^{a} t^{b} \mid(a, b) \in \mathbb{N} \mathcal{B} \cap\left(\mathbb{R}_{+} \mathcal{B}\right)^{o}\right\}\right),
$$

where $\mathcal{B}=\left\{\left(w_{1}, 1\right), \ldots,\left(w_{r}, 1\right)\right\}$ and $\left(\mathbb{R}_{+} \mathcal{B}\right)^{\circ}$ is the interior of $\mathbb{R}_{+} \mathcal{B}$ relative to aff $\left(\mathbb{R}_{+} \mathcal{B}\right)$, the affine hull of $\mathbb{R}_{+} \mathcal{B}$. In our case, aff $\left(\mathbb{R}_{+} \mathcal{B}\right)=\mathbb{R}^{n+1}$.

The next theorem complements a result of (Brennan et al. 2008). In loc. cit. a somewhat different expression for the canonical module and $a$-invariant are shown. Our expressions are simpler because they only involve the vertices of a certain polytope, while in (Brennan et al. 2008) some other parameters are involved.

THEOREM 4.1. Let $\mathcal{C}$ be a clutter with incidence matrix $A$, let $v_{1}, \ldots, v_{q}$ be the columns of $A$, and let $w_{1}, \ldots, w_{r}$ be the set of all $\alpha \in \mathbb{N}^{n}$ such that $0 \leq \alpha \leq v_{i}$ for some $i$. If the system $x \geq 0 ; x A \leq \mathbf{1}$ has the integer rounding property and $\ell_{1}, \ldots, \ell_{m}$ are the non-zero vertices of $P=\{x \mid x \geq 0 ; x A \leq \mathbf{1}\}$, then the subring $S=K\left[x^{w_{1}} t, \ldots, x^{w_{r}} t\right]$ is normal, the canonical module of $S$ is given by

$$
\omega_{S}=\left(\left\{x^{a} t^{b} \mid(a, b)\left(\begin{array}{rrrrrr}
-\ell_{1} & \cdots & -\ell_{m} & e_{1} & \cdots & e_{n} \\
1 & \cdots & 1 & 0 & \cdots & 0
\end{array}\right)>0\right\}\right),
$$

and the a-invariant of $S$ is equal to $-\left(\max _{i}\left\{\left\lfloor\left|\ell_{i}\right|\right\rfloor\right\}+1\right)$. Here $\left|\ell_{i}\right|=\left\langle\ell_{i}, \mathbf{1}\right\rangle$.

Proof. Note that in Eq. (3) we regard the $\ell_{i}$ 's and $e_{i}^{\prime} s$ as column vectors. The normality of $S$ follows from Theorem 2.1. Let $P=\{x \mid x \geq 0 ; x A \leq \mathbf{1}\}$ and let $T(P)$ be its antiblocking polyhedron

$$
T(P):=\{z \mid z \geq 0 ;\langle z, x\rangle \leq 1 \text { for all } x \in P\} .
$$

By the finite basis theorem (Schrijver 1986), we can write

$$
P=\left\{z \mid z \geq 0 ;\left\langle z, w_{i}\right\rangle \leq 1 \forall i\right\}=\operatorname{conv}\left(\ell_{0}, \ell_{1}, \ldots, \ell_{m}\right),
$$

where $\ell_{1}, \ldots, \ell_{m}, \ell_{0}$ are the vertices of $P$ and $\ell_{0}=0$. Notice that the vertices of $P$ are in $\mathbb{Q}_{+}^{n}$. From Eq. (4), we readily get the equality

$$
\left\{z \mid z \geq 0 ;\left\langle z, \ell_{i}\right\rangle \leq 1 \forall i\right\}=T(P)
$$


Using Eq. (4) again and noticing that $\left\langle\ell_{i}, w_{j}\right\rangle \leq 1$ for all $i, j$, we get

$$
\mathbb{R}_{+}^{n} \cap\left(\operatorname{conv}\left(\ell_{0}, \ldots, \ell_{m}\right)+\mathbb{R}_{+}\left\{-e_{1}, \ldots,-e_{n}\right\}\right)=\left\{z \mid z \geq 0 ;\left\langle z, w_{i}\right\rangle \leq 1 \forall i\right\}
$$

Hence, using this equality and (Schrijver 1986, Theorem 9.4), we obtain

$$
\mathbb{R}_{+}^{n} \cap\left(\operatorname{conv}\left(w_{1}, \ldots, w_{r}\right)+\mathbb{R}_{+}\left\{-e_{1}, \ldots,-e_{n}\right\}\right)=\left\{z \mid z \geq 0 ;\left\langle z, \ell_{i}\right\rangle \leq 1 \forall i\right\}
$$

By (Fulkerson 1971, Theorem 8), we have the equality

$$
\operatorname{conv}\left(w_{1}, \ldots, w_{r}\right)=\mathbb{R}_{+}^{n} \cap\left(\operatorname{conv}\left(w_{1}, \ldots, w_{r}\right)+\mathbb{R}_{+}\left\{-e_{1}, \ldots,-e_{n}\right\}\right)
$$

Therefore, using Eqs. (5) and (6), we conclude the following duality:

$$
\begin{aligned}
P=\left\{x \mid x \geq 0 ;\left\langle x, w_{i}\right\rangle \leq 1 \forall i\right\} & =\operatorname{conv}\left(\ell_{0}, \ell_{1}, \ldots, \ell_{m}\right) \\
\operatorname{conv}\left(w_{1}, \ldots, w_{r}\right) & =\left\{x \mid x \geq 0 ;\left\langle x, \ell_{i}\right\rangle \leq 1 \forall i\right\}=T(P)
\end{aligned}
$$

We set $\mathcal{B}=\left\{\left(w_{1}, 1\right), \ldots,\left(w_{r}, 1\right)\right\}$. Note that $\mathbb{Z} \mathcal{B}=\mathbb{Z}^{n+1}$. From Eq. (7), it is seen that

$$
\mathbb{R}_{+} \mathcal{B}=H_{e_{1}}^{+} \cap \cdots \cap H_{e_{n}}^{+} \cap H_{\left(-\ell_{1}, 1\right)}^{+} \cap \cdots \cap H_{\left(-\ell_{m}, 1\right)}^{+}
$$

Here $H_{a}^{+}$denotes the closed halfspace $H_{a}^{+}=\{x \mid\langle x, a\rangle \geq 0\}$ and $H_{a}$ stands for the hyperplane through the origin with normal vector $a$. Notice that

$$
H_{e_{1}} \cap \mathbb{R}_{+} \mathcal{B}, \ldots, H_{e_{n}} \cap \mathbb{R}_{+} \mathcal{B}, H_{\left(-\ell_{1}, 1\right)} \cap \mathbb{R}_{+} \mathcal{B}, \ldots, H_{\left(-\ell_{m}, 1\right)} \cap \mathbb{R}_{+} \mathcal{B}
$$

are proper faces of $\mathbb{R}_{+} \mathcal{B}$. Hence, from Eq. (8), we get that a vector $(a, b)$, with $a \in \mathbb{R}^{n}, b \in \mathbb{R}$, is in the relative interior of $\mathbb{R}_{+} \mathcal{B}$ if and only if the entries of $a$ are positive and $\left\langle(a, b),\left(-\ell_{i}, 1\right)\right\rangle>0$ for all $i$. Thus, the required expression for $\omega_{S}$, i.e., Eq. (3), follows using the normality of $S$ and the Danilov-Stanley formula given in Eq. (2).

It remains to prove the formula for $a(S)$, the $a$-invariant of $S$. Consider the vector $\left(\mathbf{1}, b_{0}\right)$, where $b_{0}=\max _{i}\left\{\left\lfloor\left|\ell_{i}\right|\right\rfloor\right\}+1$. Using Eq. (3), it is not hard to see (by direct substitution of $\left(\mathbf{1}, b_{0}\right)$ ) that the monomial $x^{\mathbf{1}} t^{b_{0}}$ is in $\omega_{S}$. Thus, from Eq. (1), we get $a(S) \geq-b_{0}$. Conversely, if the monomial $x^{a} t^{b}$ is in $\omega_{s}$, then again from Eq. (3) we get $\left\langle\left(-\ell_{i}, 1\right),(a, b)\right\rangle>0$ for all $i$ and $a_{i} \geq 1$ for all $i$, where $a=\left(a_{i}\right)$. Hence,

$$
b>\left\langle a, \ell_{i}\right\rangle \geq\left\langle\mathbf{1}, \ell_{i}\right\rangle=\left|\ell_{i}\right| \geq\left\lfloor\left|\ell_{i}\right|\right\rfloor .
$$

Since $b$ is an integer, we obtain $b \geq\left\lfloor\left|\ell_{i}\right|\right\rfloor+1$ for all $i$. Therefore, $b \geq b_{0}$, i.e., $\operatorname{deg}\left(x^{a} t^{b}\right)=b \geq b_{0}$. As $x^{a} t^{b}$ was an arbitrary monomial in $\omega_{s}$, by the formula for the $a$-invariant of $S$ given in Eq. (1), we obtain that $a(S) \leq-b_{0}$. Altogether one has $a(S)=-b_{0}$, as required. 


\section{Monomial Subrings of Cliques of Perfect Graphs}

Let $S$ be a set of vertices of a graph $G$, the induced subgraph $\langle S\rangle$ is the maximal subgraph of $G$ with vertex set $S$. A clique of a graph $G$ is a subset of the set of vertices that induces a complete subgraph. Let $G$ be a graph with vertex set $X=\left\{x_{1}, \ldots, x_{n}\right\}$. A colouring of the vertices of $G$ is an assignment of colours to the vertices of $G$ in such a way that adjacent vertices have distinct colours. The chromatic number of $G$ is the minimal number of colours in a colouring of $G$. A graph is perfect if, for every induced subgraph $H$, the chromatic number of $H$ equals the size of the largest complete subgraph of $H$. Let $S$ be a subset of the vertices of $G$. The set $S$ is called independent if no two vertices of $S$ are adjacent.

For use below we consider the empty set as a clique whose vertex set is empty. The support of a monomial $x^{a}$ is given by $\operatorname{supp}\left(x^{a}\right)=\left\{x_{i} \mid a_{i}>0\right\}$. Note that $\operatorname{supp}\left(x^{a}\right)=\emptyset$ if and only if $a=0$.

THEOREM 4.2. Let $G$ be a perfect graph and let $S=K\left[x^{\omega_{1}} t, \ldots, x^{\omega_{r}} t\right]$ be the subring generated by all square-free monomials $x^{a} t$ such that supp $\left(x^{a}\right)$ is a clique of $G$. Then, the canonical module of $S$ is given by

$$
\omega_{S}=\left(\left\{x^{a} t^{b} \mid(a, b)\left(\begin{array}{rrrrrr}
-a_{1} & \cdots & -a_{m} & e_{1} & \cdots & e_{n} \\
1 & \cdots & 1 & 0 & \cdots & 0
\end{array}\right) \geq \mathbf{1}\right\}\right),
$$

where $a_{1}, \ldots, a_{m}$ are the characteristic vectors of the maximal independent sets of $G$, and the a-invariant of $S$ is equal to $-\left(\max _{i}\left\{\left|a_{i}\right|\right\}+1\right)$.

Proof. Let $v_{1}, \ldots, v_{q}$ be the set of characteristic vectors of the maximal cliques of $G$. Note that $w_{1}, \ldots, w_{r}$ is the set of all $\alpha \in \mathbb{N}^{n}$ such that $\alpha \leq v_{i}$ for some $i$. Since $G$ is a perfect graph, by (Korte and Vygen 2000, Theorem 16.14) we have the equality

$$
P=\{x \mid x \geq 0 ; x A \leq \mathbf{1}\}=\operatorname{conv}\left(a_{0}, a_{1}, \ldots, a_{p}\right),
$$

where $a_{0}=0$ and $a_{1}, \ldots, a_{p}$ are the characteristic vectors of the independent sets of $G$. We may assume that $a_{1}, \ldots, a_{m}$ correspond to the maximal independent sets of $G$. Furthermore, since $P$ has only integral vertices, by a result of (Lovász 1972), the system $x \geq 0 ; x A \leq \mathbf{1}$ is totally dual integral, i.e., the minimum in the LP-duality equation

$$
\max \{\langle\alpha, x\rangle \mid x \geq 0 ; x A \leq \mathbf{1}\}=\min \{\langle y, \mathbf{1}\rangle \mid y \geq 0 ; A y \geq \alpha\}
$$

has an integral optimum solution $y$ for each integral vector $\alpha$ with finite minimum. In particular the system $x \geq 0 ; x A \leq \mathbf{1}$ satisfies the integer rounding property. Therefore, the result follows readily from Theorem 4.1.

For use below recall that a graph $G$ is called unmixed if all maximal independent sets of $G$ have the same cardinality. Unmixed bipartite graphs have been nicely characterized in (Villarreal 2007).

Corollary 4.3. Let $G$ be a connected bipartite graph, and let $I=I(G)$ be its edge ideal. Then, the extended Rees algebra $R\left[I t, t^{-1}\right]$ is a Gorenstein standard $K$-algebra if and only if $G$ is unmixed.

Proof. Let $\omega_{S}$ be the canonical module of $S=R\left[I t, t^{-1}\right]$. Recall that $S$ is Gorenstein if and only if $\omega_{S}$ is a principal ideal (Bruns and Herzog 1997). Since any bipartite graph is a perfect graph, the result follows using Proposition 3.1 together with the description of the canonical module given in Theorem 4.2. 


\section{ACKNOWLEDGMENTS}

This work was partially supported by CONACyT Grant 49251-F and SNI. The second author was supported by COFAA-IPN.

\section{RESUMO}

Seja $\mathcal{C}$ uma desordem (família de Sperner) e seja $A$ sua matriz de incidência. Se o sistema linear $x \geq 0 ; x A \leq \mathbf{1}$ tem a propriedade do arredondamento inteiro, fornecemos a descrição do módulo canônico e do $a$-invariante de certos subaneis monomiais associados a $\mathcal{C}$. Se a desordem é um grafo conexo, descreve-se quando o supra-mencionado sistema linear tem a propriedade do arredondamento inteiro em termos combinatórios e algébricos, usando a teoria dos grafos e a teoria das álgebras de Rees. Como consequência, mostra-se que a álgebra de Rees estendida do ideal de arestas de um grafo bipartido é um anel de Gorenstein se e somente se o grafo é de altura pura.

Palavras-chave: módulo canônico, $a$-invariante, ideal normal, grafo perfeito, cliques maximais, álgebra de Rees, anel de Erhart, propriedade do arredondamento inteiro.

\section{REFERENCES}

Brennan JP, Dupont LA And Villarreal RH. 2008. Duality, a-invariants and canonical modules of rings arising from linear optimization problems. Bull Math Soc Sci Math Roumanie (N.S.) 51: 279-305.

Bruns W and Herzog J. 1997. Cohen-Macaulay Rings. Cambridge University Press, Cambridge. Revised Edition.

DANILOV VI. 1978. The geometry of toric varieties. Russian Math Surveys 33: 97-154.

DUPONT LA AND ViLLARREAL RH. 2010. Edge ideals of clique clutters of comparability graphs and the normality of monomial ideals. Math Scand 106: 88-98.

Escobar C, Martínez-Bernal J and Villarreal RH. 2003. Relative volumes and minors in monomial subrings. Linear Algebra Appl 374: 275-290.

FULKERSON DR. 1971. Blocking and anti-blocking pairs of polyhedra. Math Programming 1: 168-194.

Gitler I, Reyes E AND Villarreal RH. 2009. Blowup algebras of square-free monomial ideals and some links to combinatorial optimization problems. Rocky Mountain J Math 39: 71-102.

Herzog J, Simis A And Vasconcelos WV. 1991. Arithmetic of normal Rees algebras. J Algebra 143: 269-294.

Hochster M. 1972. Rings of invariants of tori, Cohen-Macaulay rings generated by monomials, and polytopes. Ann of Math 96: 318-337.

Korte B And Vygen J. 2000. Combinatorial Optimization Theory and Algorithms. Springer-Verlag.

LovÁsz L. 1972. Normal hypergraphs and the perfect graph conjecture. Discrete Math 2: 253-267.

SChriJVER A. 1986. Theory of Linear and Integer Programming. J Wiley \& Sons, New York.

Simis A, Vasconcelos WV And Villarreal RH. 1994. On the ideal theory of graphs. J Algebra 167: 389-416. 
Simis A, VASCONCELOS WV AND VillarReal RH. 1998. The integral closure of subrings associated to graphs. J Algebra 199: 281-289.

Sturmfels B. 1996. Gröbner Bases and Convex Polytopes. University Lecture Series 8. Am Math Soc, Rhode Island.

Valencia C and Villarreal RH. 2003. Canonical modules of certain edge subrings. European J Combin 24 : $471-487$.

VASCONCELOS WV. 2005. Integral Closure. Springer Monographs in Mathematics, Springer, New York.

Villarreal RH. 2001. Monomial Algebras. Monographs and Textbooks in Pure and Applied Mathematics 238. Marcel Dekker, New York.

VillarReAL RH. 2005. Normality of semigroups with some links to graph theory. Discrete Math 302: 267-284.

ViLLARREAL RH. 2007. Unmixed bipartite graphs. Rev Colombiana Mat 41: 393-395. 